\title{
Necessidade de saúde insatisfeita no Brasil: uma investigação sobre a não procura de atendimento
}

\author{
Unmet health care needs in Brazil: an investigation \\ about the reasons for not seeking health care
}

Rafael Guerreiro Osorio ${ }^{1}$

Luciana Mendes Santos Servo ${ }^{1}$

Sérgio Francisco Piola ${ }^{1}$

${ }^{1}$ Diretoria de Estudos e Políticas Sociais, Instituto de Pesquisa Econômica Aplicada, Ipea. SBS Qd 01 Bloco J. 70076-900 Brasília DF. rafael.osorio@ipea.gov.br
Abstract Herein, unmet health care needs, defined by the authors as the situation when an individual feels the need but does not seek healthcare, are studied from the data of the health questionnaires of the 1998, 2003 and 2008 rounds of the National Household Sampling Survey (Pnad). From 1998 to 2008, the percentage of the population with healthcare needs during the two weeks prior to the interview did not change, remaining at around $17 \%$, whilst the share with unmet healthcare needs fell from 3.5\% to $2.9 \%$. There were also changes in the reasons chosen by the interviewees to justify why they did not seek healthcare. The percentage of those who said they could not afford it decreased - though it is still the reason given most frequently - thereby boosting the percentages of those alleging problems with the healthcare system, such as long waiting times and a lack of professionals and services. People with less income or schooling, residing in the poorer regions of the country or in rural areas, blacks, males, adults, workers, people living with other people with unmet healthcare needs or that have not been to an appointment with a doctor in the last year, and those without health plans, are less likely to seek healthcare and therefore stand a higher chance of having unmet healthcare needs. Key word Unmet healthcare needs
Resumo Neste trabalho, a necessidade de saúde insatisfeita, definida pelos autores como a situação em que o indivíduo sente necessidade, mas não procura atendimento de saúde, é estudada a partir dos dados do suplemento de saúde das rodadas de 1998, 2003 e 2008 da Pesquisa Nacional por Amostra de Domicílios (Pnad). Constata-se que, de 1998 a 2008, a parcela da população brasileira com necessidade de saúde durante as duas semanas anteriores à pesquisa se manteve em torno de $17 \%$, enquanto a parcela com necessidade de saúde insatisfeita caiu de 3,5\% para 2,9\%. Também mudou o perfil dos motivos que as pessoas escolhem para justificar não terem procurado atendimento de saúde. A porcentagem dos que alegavam falta de dinheiro se reduziu, embora ainda seja o motivo mais escolhido, aumentando as porcentagens dos que alegam limitações e falhas do sistema de saúde, como o tempo de espera e a falta de profissionais ou serviços. Baixa renda ou escolaridade, residência nas regiões mais pobres do país ou em área rural, ser negro, ser homem, ser adulto, estar ocupado, residir com outras pessoas com necessidade insatisfeita, não realizar consultas médicas há pelo menos um ano e não ter plano de saúde são, dentre outras, características que aumentam a propensão a não satisfazer a necessidade de saúde pela busca de atendimento.

Palavra-chave Necessidade de saúde insatisfeita 


\section{Introdução}

No Brasil, a saúde tem o status de direito social. Para garanti-la, a Constituição estabelece que o Sistema Único de Saúde (SUS) tenha acesso universal e atendimento integral. A realidade, porém, oferece uma série de obstáculos à consecução desses princípios. Muitos brasileiros, por motivos diversos, não procuram atendimento de saúde ao sentirem necessidade. Atingir essa parcela da população é um desafio para o SUS.

Existem várias definições de "necessidade de saúde". Em uma acepção ampliada, envolveria não apenas as necessidades relacionadas à assistência à saúde, mas também as derivadas de outras dimensões. No Brasil, segundo a revisão da bibliografia feita por Campos $^{1}$ e resumida por Campos e Mishima ${ }^{2}$, os conceitos de necessidade de saúde empregados nos estudos poderiam ser agrupados em "abstratos" e "concreto-operacionais". Em seu sentido abstrato, as necessidades estariam relacionadas a aspectos históricos e sociais, envolvendo as dimensões do direito à saúde, as necessidades relacionadas à própria garantia de sobrevivência do homem e aquelas que ultrapassam essa dimensão, como os elementos relacionados à determinação social da saúde. Em sua dimensão concreto-operacional, as necessidades de saúde são aquelas estabelecidas por quem organiza a assistência, o planejamento em saúde. Estão relacionadas à definição do objeto de atenção à saúde ${ }^{1-4}$.

$\mathrm{Na}$ literatura internacional, existem várias definições de necessidades de saúde. Referência freqüente é a proposta por Bradshaw ${ }^{5}$, a qual pode ser alinhada a uma perspectiva situada no campo concreto-operacional, principalmente por sua aproximação prática à realidade daqueles que trabalham com serviços de saúde. Para esse autor, as necessidades em saúde estão referidas às necessidades de atenção à saúde e são classificadas como percebidas (relacionadas ao campo das expectativas ou desejos), expressas (vocalizadas na demanda ou no uso dos serviços de saúde), normativas (relacionadas à avaliação por profissionais de saúde) e comparativas (aqueles que são similares entre pessoas com as mesmas características sócio-demográficas).

A necessidade de saúde seria considerada insatisfeita quando o indivíduo ou a população não recebe um serviço considerado necessário para a solução de um problema. Assim, nessa conceituação, a necessidade de saúde insatisfeita estaria relacionada ao processo de acesso aos serviços, que incluiria as necessidades percebidas, mas que não são convertidas em necessidades expressas (demanda) $)^{6}$.

Muitos estudos sobre necessidades de saúde insatisfeitas ${ }^{6-15}$ se baseiam em dados levantados por pesquisas domiciliares amostrais que investigam se, dentro de um período de referência, o entrevistado sentiu necessidade e não obteve o serviço adequado ou se não procurou assistência à saúde. São perguntadas, também, quais as causas da não satisfação da necessidade. O serviço não obtido pode estar especificado, como consulta médica, ou ser mais amplo, como consulta médica ou tratamento de saúde, podendo haver perguntas complementares sobre consultas odontológicas. Algumas pesquisas investigam em maior detalhe certas causas comuns da insatisfação da necessidade, como não poder pagar pelo serviço, não receber ou não poder comprar medicamentos prescritos, ou ter que adiar o tratamento pela indisponibilidade ou inacessibilidade do serviço necessário.

A análise da utilização de serviços de saúde no Brasil já foi objeto de diversos estudos ${ }^{16-17}$, mas a necessidade insatisfeita pela não procura de atendimento de saúde permanece inexplorada. Este trabalho visa a preencher esta lacuna no conhecimento, dedicando-se à investigação desse tipo particular de insatisfação da necessidade. Assim, alinha-se a um conjunto de estudos internacionais que abordam essa dimensão da necessidade $^{6-15}$. A preocupação é com o grupo populacional que não procurou o sistema de saúde mesmo quando sentiu que necessitava de atendimento. No caso do presente trabalho, a avaliação da satisfação da necessidade de saúde está referenciada ao nível mais elementar: a procura por assistência. Por causa desta concepção restrita, as estimativas de necessidade de saúde insatisfeita, que serão trabalhadas neste artigo, devem ser consideradas como o nível mínimo de necessidade insatisfeita, visto que, mesmo para aqueles que procuraram atendimento, a necessidade pode não ter sido satisfeita, se avaliada sobre outros aspectos, devido a problemas de suficiência, qualidade e efetividade da atenção recebida. Se esses aspectos fossem considerados, a magnitude das necessidades de saúde insatisfeitas seria maior.

O trabalho será desenvolvido dentro do campo conceitual do modelo comportamental proposto por Andersen ${ }^{18-19}$. Ainda que originalmente desenvolvido no campo da utilização de serviços, esse modelo é considerado adequado para a predição de necessidades de serviços de saúde não satisfeitas ${ }^{6,20-21}$. Por esse modelo, os preditores 
do uso de serviços de saúde podem ser classificados em: fatores de predisposição (aqueles que afetam a predisposição para o uso dos serviços, relacionados às características sócio-demográfi$\cos$ ), fatores capacitantes (relacionados à disponibilidade de meios que permitam a obtenção de cuidados à saúde) e fatores relacionados às necessidades (gravidade da doença, estado de saúde, percepção das pessoas e morbidade $)^{6,20-21}$.

\section{Métodos}

Os suplementos de saúde da Pesquisa Nacional por Amostra de Domicílios (Pnad) permitem avaliar a satisfação de algumas necessidades de saúde. Para estudar esse tema a partir desta pesquisa é preciso usar as respostas dadas a questões específicas constantes dos questionários suplementares de saúde que foram a campo nos anos de 1998, 2003 e 2008.

As informações necessárias para identificar a parcela da população com necessidade de saúde são obtidas no bloco de questões sobre a utilização de serviços de saúde nas duas semanas anteriores à entrevista. Assim, o período de identificação da necessidade de saúde tem amplitude igual a do período de coleta mais duas semanas. Essa é uma diferença marcante em relação às pesquisas de outros países ${ }^{6-15}$, que adotam períodos de referência maiores, geralmente de um ano.

Ademais, a PNAD, diferentemente de pesquisas amostrais realizadas em outros países, não possui uma pergunta direta sobre se a pessoa sentiu alguma necessidade de saúde. Em vista disso, essa informação tem que ser inferida a partir das respostas a duas perguntas. A primeira é a que abre o bloco de questões sobre a utilização de serviços: Procurou algum lugar, serviço ou profissional de saúde para atendimento relacionado à própria saúde nas duas últimas semanas? ${ }^{22}$. Segundo o IBGE, o propósito da pergunta é investigar a procura por algum tipo de atendimento relacionado à saúde ${ }^{22}$.

Essa pergunta é aplicável a todos os membros do grupo doméstico. Caso a resposta seja afirmativa, o questionário prossegue levantando informações que caracterizam a necessidade de saúde daqueles que procuraram atendimento e os serviços recebidos para supri-la: qual o local procurado, que tipo de serviço ou profissional foi usado, como foi o atendimento e outros detalhes. A segunda, só é preenchida nos casos em que a resposta ao quesito referente à procura por serviços de saúde tenha sido negativa. Nestes ca- sos, o entrevistador passa direto ao quesito que identifica o Motivo pelo qual não procurou serviço de saúde nas duas últimas semanas ${ }^{22}$. A lista de motivos possíveis de não procura por atendimento é predefinida: o primeiro é não houve necessidade $^{22}$; e os demais (listados na Tabela 1 com suas definições) são justificativas para a não procura de um serviço ou profissional. Assim, a não ser quando a resposta tenha sido não houve necessidade, para os casos em que a pessoa procurou algum serviço de saúde ou que não procurou mais declarou outros motivos, assume-se que houve necessidade. O grupo de pessoas que tiveram necessidade de saúde é então dividido em dois subgrupos: os dos que em face à necessidade procuraram um serviço, e os que não procuraram e escolheram um motivo, exceto não ter tido necessidade, como justificativa da não procura.

Para aqueles que sentiram necessidade, mas não procuraram algum serviço de saúde, havia uma lista pré-definida de motivos que eles poderiam escolher para justificar a não procura. Para as análises realizadas nesse artigo, excetuando-se a categoria outros motivos, os demais foram agrupados em dois subgrupos de necessidades insatisfeitas por: (i) motivos individuais e (ii) motivos relacionados ao sistema; (iii) outros motivos. Como motivos individuais foram incluídos: falta de dinheiro, dificuldade de transporte, falta de acompanhante e ignorância sobre o direito à saúde. Os motivos relacionados ao sistema foram: o local de atendimento era distante ou de difícil acesso, horário incompatível, o atendimento é muito demorado, o estabelecimento não possuía especialista compatível; não gostava dos profissionais do estabelecimento e greve nos serviços de saúde (Tabela 1).

A despeito da relevância de se constatar que uma parcela da população não satisfaz sua necessidade de saúde procurando atendimento e da descrição dos motivos apresentados para não fazê-lo, para a política de saúde é importante saber se o perfil dessa população apresenta características distintas em relação à parcela da população que sentiu necessidade e buscou atendimento. Para tal fim, uma série de características - e suas distribuições - foi investigada para a população brasileira e para as seguintes subpopulações: com necessidade de saúde; que não satisfez a necessidade; que não satisfez por motivos individuais; que não satisfez por motivos relacionados ao sistema; que não satisfez por outros motivos.

Para essa análise foram selecionadas como variáveis aquelas relacionadas aos fatores de pre- 
Tabela 1. Distribuição relativa da população com necessidade de saúde insatisfeita segundo motivos de não procurar atendimento. Brasil ${ }^{(1)}, 1998,2003$ e 2008

\begin{tabular}{|c|c|c|c|c|}
\hline \multirow{2}{*}{ Motivo } & \multirow{2}{*}{ Definição } & \multicolumn{3}{|c|}{$\begin{array}{c}\text { \% da população com } \\
\text { necessidade insatisfeita } \\
(2)\end{array}$} \\
\hline & & 1998 & 2003 & 2008 \\
\hline a) Não tinha dinheiro & $\begin{array}{l}\text { Quando a pessoa não procurou serviço de saúde } \\
\text { porque não tinha dinheiro suficiente para o } \\
\text { deslocamento e/ou efetuar o pagamento }\end{array}$ & 32,5 & 23,7 & 20,9 \\
\hline $\begin{array}{l}\text { b) O local de atendimento era } \\
\text { distante ou de difícil acesso }\end{array}$ & $\begin{array}{l}\text { Quando a pessoa não procurou serviço de saúde } \\
\text { porque o local de atendimento era distante de } \\
\text { sua residência ou de difícil acesso }\end{array}$ & 11,7 & 7,5 & 11,0 \\
\hline $\begin{array}{l}\text { c) Dificuldade de conseguir } \\
\text { transporte }\end{array}$ & $\begin{array}{l}\text { Quando a pessoa não procurou serviço de } \\
\text { sauìde devido à dificuldade de conseguir } \\
\text { transporte, inclusive por motivo de greve nos } \\
\text { transportes coletivos }\end{array}$ & 5,2 & 5,2 & 5,6 \\
\hline d) Horário incompatível & $\begin{array}{l}\text { Quando a pessoa não procurou serviço de saúde } \\
\text { porque o horário de funcionamento do } \\
\text { estabelecimento ao qual recorreria era } \\
\text { incompatível com o horário em que poderia ir }\end{array}$ & 9,7 & 12,6 & 14,0 \\
\hline $\begin{array}{l}\text { e) O atendimento é muito } \\
\text { demorado }\end{array}$ & $\begin{array}{l}\text { Quando a pessoa não procurou serviço de saúde } \\
\text { por julgar que o atendimento dos serviços de } \\
\text { saúde era muito demorado, inclusive quando } \\
\text { indicou como causa da demora a necessidade } \\
\text { de marcação prévia de consulta, ficar em fila ou } \\
\text { chegar cedo para pegar senha }\end{array}$ & 13,0 & 18,3 & 17,9 \\
\hline $\begin{array}{l}\text { f) O estabelecimento não } \\
\text { possuía o especialista } \\
\text { compatível com suas } \\
\text { necessidades }\end{array}$ & $\begin{array}{l}\text { Quando a pessoa não procurou serviço de saúde } \\
\text { porque achava ou tinha informação de que no } \\
\text { estabelecimento de saúde ao qual poderia } \\
\text { recorrer não havia o especialista que necessitava }\end{array}$ & 4,2 & 4,8 & 9,4 \\
\hline $\begin{array}{l}\text { g) Achou que não tinha } \\
\text { direito }\end{array}$ & $\begin{array}{l}\text { Quando a pessoa não procurou serviço de saúde } \\
\text { porque achou que não tinha direito ao } \\
\text { atendimento que necessitava como, por } \\
\text { exemplo, por achar que o estabelecimento só } \\
\text { atenderia moradores da área ou que tivessem } \\
\text { plano de saúde }\end{array}$ & 0,3 & 0,5 & 0,4 \\
\hline $\begin{array}{l}\text { h) Não tinha quem o(a) } \\
\text { acompanhasse }\end{array}$ & $\begin{array}{l}\text { Quando a pessoa não procurou serviço de saúde } \\
\text { porque não tinha quem lhe fizesse companhia e } \\
\text { não podia ir sozinha devido à idade, dificuldade } \\
\text { de se locomover sozinha, gravidade do } \\
\text { problema de saúde ou razões psicológicas, } \\
\text { emocionais ou de simples constrangimento }\end{array}$ & 2,5 & 2,1 & 2,0 \\
\hline $\begin{array}{l}\text { i) Não gostava dos } \\
\text { profissionais do } \\
\text { estabelecimento }\end{array}$ & $\begin{array}{l}\text { Quando a pessoa não procurou serviço de saúde } \\
\text { porque não gostava dos profissionais do } \\
\text { estabelecimento }\end{array}$ & & 3,6 & 2,7 \\
\hline j) Greve nos serviços de saúde & $\begin{array}{l}\text { Quando a pessoa não procurou serviço de saúde } \\
\text { porque os serviços de saúde estavam paralisados } \\
\text { por motivo de greve dos profissionais de saúde }\end{array}$ & & 0,2 & 0,2 \\
\hline k) Outro motivo & $\begin{array}{l}\text { Quando a pessoa não procurou serviço de saúde } \\
\text { por motivo que não se enquadrava nos itens } \\
\text { anteriores, como, por exemplo, por falta de } \\
\text { cartão, carteira de plano de saúde com validade } \\
\text { vencida, carência de plano de saúde }\end{array}$ & 20,5 & 21,4 & 15,9 \\
\hline
\end{tabular}

População com necessidade insatisfeita

Fonte: IBGE, Pesquisa Nacional por Amostra de Domicílios em microdados. (1) Exceto as áreas rurais do Acre, Amapá, Amazonas, Pará, Rondônia e Roraima. (2) Em 1998 e em 2003 o motivo era ignorado em 0,3\% dos casos; em 2008 foi feita imputaçaPo estatìstica de motivos. 
disposição (idade, sexo, cor ou raça, escolaridade; condição na ocupação); fatores capacitantes (renda; situação de domicílio; região; cadastro na Saúde da Família (SF) e adesão a plano de saúde); variáveis relacionadas à saúde/à necessidade (auto-avaliação do estado de saúde; mobilidade limitada; doença crônica). Cabe destacar que a informação sobre o cadastro na SF só está disponível no suplemento da Pnad de 2008.

Algumas variáveis usadas no perfil foram derivadas a partir de variáveis originais, e outras tiveram que ser ajustadas, como a de realização de consultas médicas. Neste caso, utilizamos como referência as consultas no ano anterior, excluídas aquelas realizadas nas duas últimas semanas.

Além disso, no questionário suplementar de saúde são investigadas as seguintes doenças crônicas: a) doença de coluna ou costas; b) artrite ou reumatismo; c) câncer; d) diabetes; bronquite ou asma; e) hipertensão; f) doença do coração; g) insuficiência renal crônica; h) depressão; i) tuberculose; $\mathrm{j}$ ) tendinite ou tenossinovite; $\mathrm{k}$ ) cirrose (em 2003 e em 2008, as doenças crônicas precisavam ter sido diagnosticadas por médico ou profissional de saúde). Uma pessoa que tivesse qualquer uma dessas foi marcada como tendo doença crônica.

A seção de perguntas sobre mobilidade física foi ampliada de 1998 a 2008, e aqui foram consideradas apenas as perguntas que constavam no questionário suplementar de 1998, que investigavam se a pessoa tinha dificuldade para: a) alimentar-se, tomar banho ou ir ao banheiro; b) correr, levantar objetos pesados, praticar esportes ou realizar trabalhos pesados; c) empurrar mesa ou realizar consertos domésticos; d) subir ladeira ou escada; e) abaixar-se, ajoelhar-se ou curvar-se; f) andar mais do que um quilômetro; g) andar cerca de 100 metros. Considerou-se com limitação da mobilidade física a pessoa que respondeu enfrentar alguma dificuldade para realizar ao menos uma dessas ações (as respostas possíveis eram: não consegue, tem grande dificuldade; tem pequena dificuldade; e não tem dificuldade).

Para modelar a necessidade de saúde insatisfeita, partiu-se da constatação de que, na forma definida neste artigo e passível de ser investigada a partir da Pnad, a necessidade insatisfeita pode servir como proxy para a falta ou restrições de acesso ao sistema de saúde, na linha de estudos realizados em outros países. Concebida como uma variável latente, essa dimensão da falta ou restrições de acesso é dada pela probabilidade de não buscar os serviços de saúde ao ter uma ne- cessidade. Sendo conhecida a não procura de atendimento por parte da população com necessidade de saúde, em um período limitado de tempo, é possível estimar a probabilidade desse evento.

Essa probabilidade, por sua vez, depende das dos indivíduos, que não são idênticas e que dependem de uma série de características. Cada indivíduo possui sua própria probabilidade, e nos interessa destacar a parte não explicável que cabe a suas idiossincrasias (resíduo) da explicável que se deve ao pertencimento a grupos. Saber quais características específicas a influenciam, e quanto, permite delimitar grupos de indivíduos em maior risco de insatisfação de necessidade de saúde. Por exemplo, em relação a uma mulher de mesma idade, sexo, residindo na mesma área/ região, com as mesmas características de saúde, na mesma faixa de renda e com a mesma condição de ocupação - interessa saber se, e quanto, ser homem afeta a probabilidade de insatisfação da necessidade de saúde.

Essa abordagem metodológica leva quase automaticamente à escolha de um modelo linear para variáveis dependentes dicotômicas para o caso da insatisfação da necessidade de saúde. Optamos pelo logit, pela simplicidade de interpretação em termos de razões de chances. O conjunto de variáveis independentes são aquelas relacionadas aos fatores de predisposição, capacitantes e às necessidades (Tabela 2).

As categorias de base escolhidas foram as que se caracterizaram pelo baixo risco no perfil univariado: ter de zero a quatro anos de idade, ser do sexo feminino, não ter ocupação, ser branco ou amarelo, ter educação superior, morar em área urbana, na região Sul, fazer parte dos 20\% mais ricos, auto-avaliar sua saúde como regular, boa ou ótima, ter suspenso as atividades nas duas semanas anteriores, ter ficado acamado nesse período, ter consultado médico no ano anterior (exceto nas duas semanas anteriores), não ter doença crônica, não ter limitação de mobilidade física, ter um plano de saúde, e ter sido o grupo doméstico cadastrado pela ESF (Equipe de Saúde da Família).

Às características individuais ou fatores de predisposição foi acrescida a presença de outras pessoas com necessidade de saúde no grupo doméstico. Essa característica é representada por três variáveis dicotômicas. A base é nenhum outro morador teve necessidade; as demais são: outros moradores tiveram necessidade, mas não procuraram o serviço de saúde; outros moradores tiveram necessidade e ao menos um não procurou (essa categoria requer haver ao menos três pessoas com necessidade no grupo doméstico: independentemente da 
Tabela 2. Distribuição relativa de características selecionadas na população total e em subpopulações. Brasil ${ }^{(1)}, 2008$

\begin{tabular}{|c|c|c|c|c|c|c|}
\hline \multirow{5}{*}{ Característica } & \multicolumn{6}{|c|}{ \% da população } \\
\hline & \multirow{4}{*}{ Total } & \multicolumn{5}{|c|}{ Com necessidade de saúde } \\
\hline & & \multirow{3}{*}{ Total } & \multicolumn{4}{|c|}{ Insatisfeita } \\
\hline & & & \multirow[t]{2}{*}{ Total } & \multicolumn{3}{|c|}{ Categorias de motivos } \\
\hline & & & & Circ. indiv. & Circ. sist. & Outros \\
\hline \multicolumn{7}{|l|}{ Idade } \\
\hline 0 a 4 anos & 7,2 & 8,5 & 3,8 & 4,2 & 3,6 & 4,0 \\
\hline 5 a 14 anos & 17,6 & 11,2 & 9,0 & 11,6 & 8,3 & 7,2 \\
\hline 15 a 24 anos & 17,6 & 12,7 & 11,7 & 11,1 & 12,1 & 11,3 \\
\hline 25 a 44 anos & 30,5 & 29,1 & 33,1 & 29,1 & 35,5 & 32,6 \\
\hline 45 a 54 anos & 11,7 & 14,9 & 16,8 & 16,1 & 17,3 & 16,6 \\
\hline 55 a 64 anos & 7,7 & 11,0 & 11,7 & 11,0 & 12,0 & 12,0 \\
\hline 65 a 74 anos & 4,7 & 7,5 & 8,1 & 9,1 & 6,9 & 9,8 \\
\hline 75 ou mais & 2,9 & 5,1 & 5,7 & 7,8 & 4,3 & 6,6 \\
\hline \multicolumn{7}{|l|}{ Sexo } \\
\hline Feminino & 51,3 & 59,4 & 54,4 & 55,0 & 57,1 & 45,8 \\
\hline Masculino & 48,7 & 40,6 & 45,6 & 45,0 & 42,9 & 54,2 \\
\hline \multicolumn{7}{|l|}{ Raça } \\
\hline Brancos e amarelos & 49,3 & 49,8 & 36,1 & 33,5 & 36,3 & 39,4 \\
\hline Pardos, pretos e indígenas & 50,7 & 50,2 & 63,9 & 66,5 & 63,7 & 60,6 \\
\hline \multicolumn{7}{|l|}{ Educação $^{(2)}$} \\
\hline Nem elementar & 34,3 & 36,9 & 54,1 & 63,4 & 50,1 & 50,6 \\
\hline Elementar & 19,4 & 19,4 & 18,9 & 16,5 & 19,3 & 21,6 \\
\hline Fundamental & 14,5 & 13,4 & 11,4 & 8,9 & 12,7 & 11,5 \\
\hline Médio & 23,7 & 21,9 & 12,8 & 9,3 & 14,5 & 13,3 \\
\hline Superior & 8,2 & 8,4 & 2,9 & 1,9 & 3,4 & 3,1 \\
\hline \multicolumn{7}{|l|}{ Trabalho } \\
\hline Ocupados & 48,4 & 46,3 & 52,1 & 45,0 & 55,7 & 52,7 \\
\hline Desocupados/inativos & 51,6 & 53,7 & 47,9 & 55,0 & 44,3 & 47,3 \\
\hline \multicolumn{7}{|l|}{ Renda } \\
\hline $20+$ pobres & 20,0 & 18,8 & 31,9 & 38,8 & 29,7 & 27,2 \\
\hline $20-40$ & 20,0 & 19,0 & 23,7 & 23,0 & 24,7 & 21,7 \\
\hline $40-60$ & 20,0 & 20,8 & 22,2 & 22,0 & 22,0 & 23,0 \\
\hline $60-80$ & 20,0 & 19,9 & 14,4 & 11,8 & 15,2 & 16,1 \\
\hline $20+$ ricos & 20,0 & 21,4 & 7,9 & 4,4 & 8,3 & 12,0 \\
\hline \multicolumn{7}{|l|}{ Situação do domicílio } \\
\hline Urbana & 84,9 & 86,0 & 76,0 & 67,2 & 78,6 & 82,5 \\
\hline Rural & 15,1 & 14,0 & 24,0 & 32,8 & 21,4 & 17,5 \\
\hline \multicolumn{7}{|l|}{ Região } \\
\hline Norte & 6,6 & 5,9 & 8,6 & 7,6 & 9,8 & 6,9 \\
\hline Nordeste & 29,0 & 28,6 & 45,7 & 48,7 & 44,6 & 44,4 \\
\hline Sudeste & 42,1 & 43,3 & 28,5 & 26,3 & 28,7 & 31,4 \\
\hline Sul & 14,9 & 15,2 & 9,3 & 9,5 & 8,8 & 10,1 \\
\hline Centro-Oeste & 7,4 & 7,1 & 7,8 & 7,9 & 8,0 & 7,2 \\
\hline \multicolumn{7}{|l|}{ Estado de saúde } \\
\hline Avaliação negativa & 3,8 & 11,6 & 18,5 & 21,6 & 16,0 & 20,4 \\
\hline Atividades suspensas & 8,4 & 32,7 & 30,0 & 28,0 & 26,4 & 43,0 \\
\hline Acamado & 3,9 & 16,6 & 15,4 & 14,4 & 13,4 & 22,6 \\
\hline Doença crônica & 31,4 & 52,1 & 54,4 & 55,0 & 53,6 & 55,4 \\
\hline Mobilidade limitada & 22,5 & 42,1 & 53,6 & 55,8 & 52,2 & 54,2 \\
\hline \multicolumn{7}{|l|}{ Acesso à saúde } \\
\hline Consulta médica & 66,4 & 81,5 & 66,8 & 63,7 & 69,5 & 64,1 \\
\hline Cadastro ESF & 51,3 & 52,8 & 56,9 & 55,3 & 56,4 & 60,9 \\
\hline Plano de saúde & 25,8 & 29,7 & 9,4 & 5,8 & 9,3 & 15,2 \\
\hline População & 180.651 .126 & 31.557 .598 & 5.209 .625 & 1.503 .353 & 2.724 .771 & 981.501 \\
\hline
\end{tabular}

Fonte: IBGE, Pesquisa Nacional por Amostra de Domicílios em microdados. (1) Exceto as áreas rurais do Acre, Amapá, Amazonas, Pará, Rondônia e Roraima. (2) Para pessoas de zero a 24 anos que não eram pessoa de referência ou cônjuge no grupo doméstico, considerou-se o nível educacional da pessoa de referência. 
procura por parte do indivíduo em questão, há ao menos outra pessoa que procurou e ao menos outra que não procurou); outros moradores tiveram necessidade e procuraram.

O modelo foi estimado com o pacote estatístico Stata buscando considerar o desenho amostral da Pnad (com a variável v4617 definindo os estratos, a v4618 delimitando as unidades primárias de amostragem, e os pesos dando a probabilidade de seleção de cada observação). Foram consideradas 64.283 observações (que expandidas representam 31,5 milhões de pessoas) distribuídas em 785 estratos e 5626 unidades primárias (desenho com 4.841 graus de liberdade); sua estatística $\mathrm{F}(34 ; 4808)$ foi estimada em 202,5, atestando a significância global do modelo para qualquer nível convencional ( $\mathrm{P}>0,0000)$. Os coeficientes do modelo são apresentados em logaritmo e já convertidos para razões de chances, junto com seus erros padrão, os $p$-valores associados à estatística de teste da hipótese de o coeficiente ser zero na população, e os valores inferior e superior do intervalo de confiança de $95 \%$ das razões de chances.

\section{Resultados}

A porcentagem da população com necessidade de saúde no período de duas semanas antes da entrevista ficou sempre próxima a $17 \%$ no período
1998-2008 (Gráfico 1). Em números absolutos, 25,2 milhões de pessoas tiveram necessidade de saúde em 1998, passando a 31,6 milhões em 2008. A variação dessa porcentagem de 1998 a 2003 é menor do que um ponto percentual, ou seja, é irrelevante apesar de estatisticamente significante.

Adotando como denominador a população total, o percentual de brasileiros com necessidade de saúde insatisfeita nas duas semanas anteriores à pesquisa foi de 3,5\%, em 1998, e 2,9\%, em 2008. Porém, o tamanho da população com necessidades de saúde insatisfeitas em 2008 continuou quase o mesmo de 1998, pouco mais de cinco milhões de pessoas.

Em relação à população com necessidade de saúde, a porcentagem de pessoas que não satisfez a necessidade via procura de atendimento caiu de $21 \%$ em 1998 a $16 \%$ em 2003, e se manteve próxima desse nível em 2008 (Gráfico 2).

Esses dados do Brasil, por causa das diferenças na forma de coletar a informação (perguntas e período de referência), não são estritamente comparáveis aos de outros países, que também não são sempre comparáveis entre si. Mesmo as estimativas de um país podem variar por causa dessas diferenças: na Dinamarca, dependendo do estudo e do conceito adotado, a necessidade de saúde insatisfeita pode variar de $1 \%$ a $4 \%$ da população ${ }^{13}$

Ainda assim, apenas a título de ilustração, pode-se mencionar que, na Europa, as porcen-

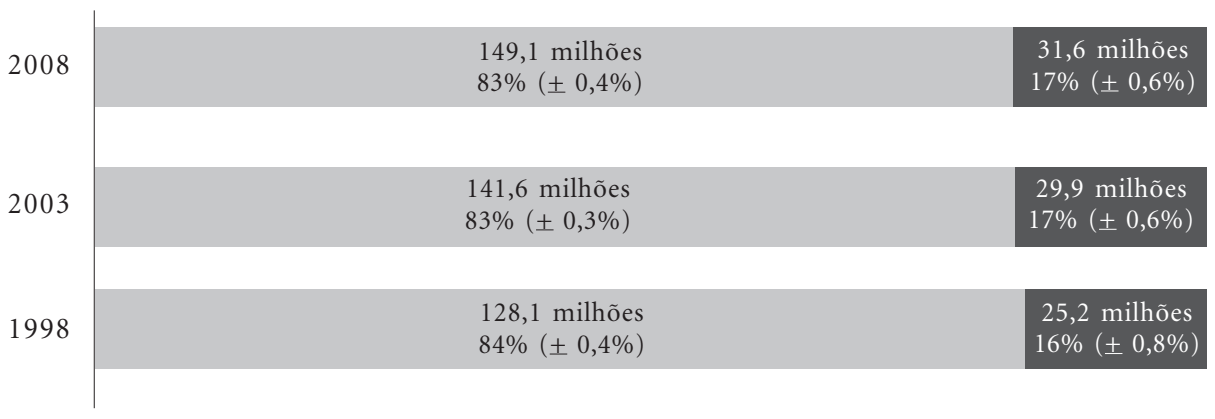

Sem necessidade $\quad$ Com necessidade

Gráfico 1. Frequência absoluta (em milhões de pessoas), relativa (em \%) e variação porcentual das estimativas da população segundo a necessidade de saúde. Brasil ${ }^{(1)}$ 1998, 2003 e 2008

Fonte: IBGE, Pesquisa Nacional por Amostra de Domicílios em microdados. (1) Exceto as áreas rurais do Acre, Amapá, Amazonas, Pará, Rondônia e Roraima. 


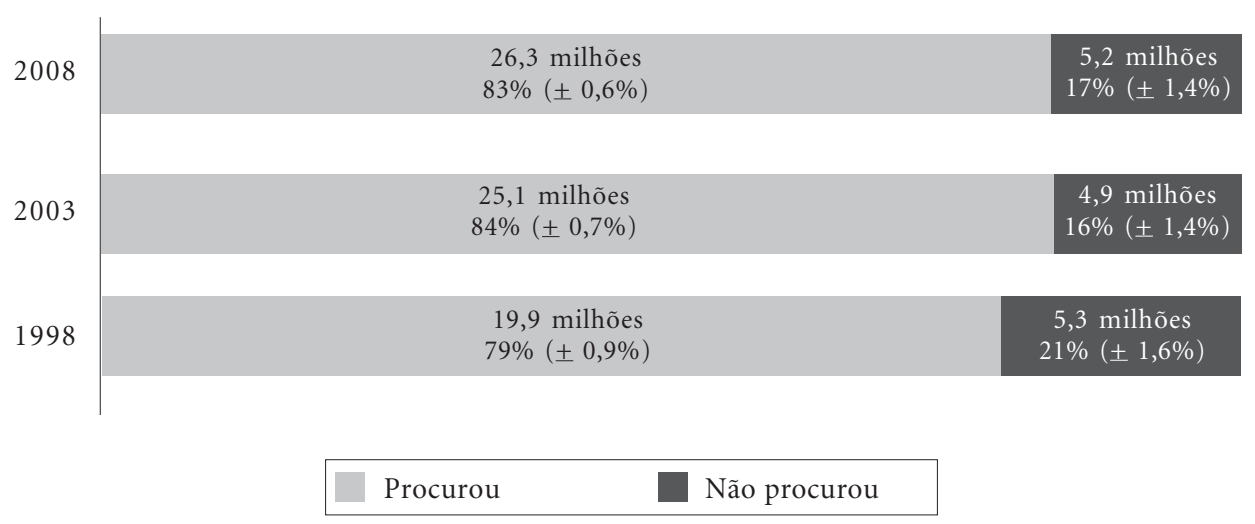

Gráfico 2. Frequência absoluta (em milhões de pessoas), relativa (em \%) e variação porcentual das estimativas de pessoas que tiveram necessidade de saúde segundo a procura de atendimento. Brasil(1), 1998, 2003 e 2008

Fonte: IBGE, Pesquisa Nacional por Amostra de Domicílios em microdados. (1) Exceto as áreas rurais do Acre, Amapá, Amazonas, Pará, Rondônia e Roraima.

tagens da população com necessidade de saúde insatisfeita variam de $1 \%$ na Dinamarca a $13 \%$ na Suécia ${ }^{13}$ Nos Estados Unidos, em 1994, aproximadamente $6 \%$ da população tinham necessidade de saúde insatisfeita (não ter obtido atenção médica ou cirurgia $)^{14}$, e incluindo na estimativa de necessidade insatisfeita todos os componentes pesquisados (acesso a medicamentos prescritos, a óculos, a tratamento odontológico, e a atenção a saúde mental ou serviços de aconselhamento), 16,1\% da população não conseguiram obter ao menos um dos serviços ou bens que consideravam necessitar. No Canadá, 12,5\% da população ${ }^{15}$ de 12 anos ou mais declarou ter tido uma necessidade de saúde insatisfeita no período 2000-01.

Sem esquecer as diferenças de conceitos e períodos, enquanto que no Brasil há tendência de diminuição da parcela da população com necessidade de saúde insatisfeita, em outros países a tendência é de aumento. Nos Estados Unidos ${ }^{14} \mathrm{e}$ no Canadá ${ }^{15}$, pesquisadores constataram, ao comparar seus resultados aos de estudos anteriores, o aumento da parcela da população com necessidade insatisfeita. No caso do Canadá, o aumento não é atribuído ao envelhecimento populacional em curso, mas a outros fatores, como as restrições pessoais de acesso (por exemplo, a falta de tempo para cuidar de si) e o grau de conhecimento dos serviços prestados pelo sistema de saúde.
No Brasil, quem não procurou atendimento, embora tivesse tido necessidade de saúde, escolheu um dos motivos apresentados na Tabela 1 para explicar o porquê de não ter procurado. A comparação das distribuições por motivos revela a redução, de 1998 a 2008, em dez pontos da porcentagem de pessoas que explicavam não ter procurado atendimento por falta de dinheiro. Esses dez pontos percentuais se distribuíram pelas porcentagens dos que justificaram não ter procurado atendimento por incompatibilidade de horário, pelo longo tempo de espera, ou pela falta de especialistas. As porcentagens dos outros motivos variaram pouco.

Agregando as categorias de motivos, nota-se que a porcentagem da população com necessidade insatisfeita apontando circunstâncias individuais como justificativa para não procurar atendimento - falta de dinheiro, dificuldade de transporte, falta de acompanhante, ou ignorância sobre o direito à saúde (motivos em negrito na Tabela 1) - caiu de 40\% em 1998 para 29\% em 2008. Esse decréscimo foi compensado pelo aumento da porcentagem dos que alegam limitações ou falhas do sistema de saúde como razão para não procurar atendimento. Essas podem ser reais ou fruto de percepções e expectativas negativas em relação ao sistema de saúde. Os entrevistados alegam não ter procurado atendimento por ser feito em local distante ou de difícil acesso, em horário incompatível, com longa es- 
pera, ou por não contar com o especialista apropriado (motivos em itálico na Tabela 1). Assim, a distribuição dos motivos de insatisfação em 1998 era mais semelhante à encontrada nos Estados Unidos, onde a insatisfação está relacionada principalmente às restrições orçamentárias, apontadas por mais da metade dos insatisfeitos $^{14}$; enquanto a de 2008 se assemelhava mais à do Canadá15, onde a insatisfação decorre majoritariamente do longo tempo de espera e da indisponibilidade dos serviços no momento em que são percebidos como necessários.

A Tabela 2 permite um esboço do perfil da população com necessidade insatisfeita. Mulheres e pessoas com mais de 44 anos - ou crianças com menos de cinco anos - têm maior probabilidade de necessitar atendimento. Mas a necessidade de saúde não distingue as pessoas segundo outras características socioeconômicas: pobres e ricos, pouco e muito educados, negros e brancos, nordestinos e sulistas, moradores do campo e da cidade, ocupados e não, todos estão sujeitos, em intensidade semelhante, a precisar de atendimento.

A propensão a satisfazer a necessidade, porém, se comporta de forma distinta. Os fatores que provocam maior necessidade de saúde parecem diminuir a propensão a não satisfazê-la. Considerando idade e sexo, tem-se que homens jovens e adultos têm menor necessidade de saúde, mas são mais propensos a não satisfazê-la. E os demais fatores socioeconômicos, a despeito de não influírem sobre a necessidade, parecem todos associados a não procura do atendimento, com a intensidade da associação variando segundo categorias de motivos.

O último bloco de características na Tabela 2 está diretamente ligado à saúde. Essas características são forte e positivamente correlacionadas à necessidade de saúde. Pessoas que auto-avaliam seu estado de saúde como ruim ou muito ruim, que tiveram que suspender suas atividades por ao menos um dia nas duas semanas anteriores por causa de problemas de saúde, ou que ficaram acamadas ao menos um dia nesse período, que fizeram ao menos uma consulta médica no ano anterior, que possuem alguma doença crônica ou têm a mobilidade física limitada, se apresentam em porcentagens bem mais altas na população com necessidade do que na população em geral.

Contudo, apenas algumas características do acesso se correlacionaram fortemente com a não satisfação das necessidades. A que mais chama a atenção é a realização de consulta médica no ano anterior: pessoas com essa característica apresen- tam uma propensão consideravelmente menor de não satisfazerem sua necessidade de saúde. O cadastramento do grupo doméstico na estratégia Saúde da Família está associado à insatisfação da necessidade. Finalmente, a posse de um plano de saúde causa uma redução considerável na propensão a não procurar atendimento.

Em geral, as distribuições das características nos subgrupos definidos pelas categorias de motivos são bem semelhantes às da população com necessidade insatisfeita. Todavia, existem algumas diferenças. As pessoas menos educadas e as mais pobres são relativamente mais freqüentes entre os que apontam circunstâncias individuais como motivo da não procura. Adultos, ocupados, mais educados, mais ricos, residentes em áreas urbanas, se manifestam em maior proporção entre os que atribuem a insatisfação de sua necessidade às circunstâncias do sistema de saúde.

Por enquanto, essas e as demais correlações devem ser encaradas com precaução, pois a análise até este ponto é univariada e prospectiva. Assim, algumas variações podem ser parcialmente devidas a fatores não-controlados, e nem tanto à característica em questão. Por exemplo, o fato de pessoas cujos grupos domésticos estão cadastrados na estratégia Saúde da Família se apresentarem em porcentagens pouco maiores na população com necessidade de saúde insatisfeita pode se dever à associação do cadastro com outras características que são fatores de risco de insatisfação (como a maior cobertura do ESF nas áreas mais carentes). Os resultados do modelo logit na Tabela 3 eliminam o problema, mostrando o efeito isolado de cada fator.

Inicialmente, percebe-se que vários fatores têm razões de chances próximas a um e, em alguns casos, são diferentes de um para níveis pouco rigorosos de significância. Doenças crônicas, por exemplo, são irrelevantes para determinar a satisfação da necessidade. Entre as características pouco relevantes figura a educação da pessoa (ou da pessoa de referência do grupo doméstico para as crianças e jovens de zero a vinte e quatro anos que não eram pessoas de referência ou cônjuges em seus grupos domésticos). Não ter ao menos educação elementar, ou apenas tê-la, aumenta a chance de não satisfazer a necessidade de saúde em relação a quem tem nível superior. Não obstante, a chance dos menos educados é apenas um pouco maior, 1,3 vezes a de quem tem educação superior.

O sexo e a raça, isoladamente, também pouco fazem variar a probabilidade de não procurar atendimento. Por exemplo, segundo o modelo, uma 
Tabela 3. Necessidade de saúde insatisfeita - resultados do modelo logit. Brasil ${ }^{(1)}, 2008$

\begin{tabular}{|c|c|c|c|c|c|c|}
\hline \multirow{2}{*}{ Característica } & \multicolumn{3}{|c|}{ Razão entre chances } & \multirow{2}{*}{$\begin{array}{c}\text { Coef. } \\
(\log -\text { razão })\end{array}$} & \multirow{2}{*}{$\begin{array}{c}\text { Erro } \\
\text { padrão }\end{array}$} & \multirow{2}{*}{ p-valor } \\
\hline & $\begin{array}{l}\text { Mínimo } \\
\text { I.C. } 95 \%\end{array}$ & Estimada & $\begin{array}{l}\text { Máximo } \\
\text { I.C. 95\% }\end{array}$ & & & \\
\hline \multicolumn{7}{|l|}{ Idade } \\
\hline 0 a 4 anos (base) & & 1,00 & & & & \\
\hline 5 a 14 anos & 1,16 & 1,30 & 1,47 & 0,2662 & 0,0604 & 0,0000 \\
\hline 15 a 24 anos & 1,27 & 1,42 & 1,59 & 0,3513 & 0,0589 & 0,0000 \\
\hline 25 a 44 anos & 1,86 & 2,07 & 2,30 & 0,7258 & 0,0535 & 0,0000 \\
\hline 45 a 54 anos & 1,68 & 1,87 & 2,08 & 0,6271 & 0,0548 & 0,0000 \\
\hline 55 a 64 anos & 1,49 & 1,67 & 1,87 & 0,5127 & 0,0568 & 0,0000 \\
\hline 65 a 74 anos & 1,71 & 1,94 & 2,21 & 0,6650 & 0,0664 & 0,0000 \\
\hline 75 ou mais & 1,81 & 2,11 & 2,46 & 0,7476 & 0,0777 & 0,0000 \\
\hline \multicolumn{7}{|l|}{ Sexo } \\
\hline Feminino (base) & & 1,00 & & & & \\
\hline Masculino & 1,13 & 1,20 & 1,27 & 0,1810 & 0,0286 & 0,0000 \\
\hline \multicolumn{7}{|l|}{ Raca } \\
\hline Brancos e amarelos (base) & & 1,00 & & & & \\
\hline Pardos, pretos e indígenas & 1,10 & 1,16 & 1,23 & 0,1475 & 0,0285 & 0,0000 \\
\hline \multicolumn{7}{|l|}{ Educação ${ }^{(2)}$} \\
\hline Nem elementar & 1,15 & 1,34 & 1,58 & 0,2959 & 0,0809 & 0,0003 \\
\hline Elementar & 1,05 & 1,23 & 1,44 & 0,2053 & 0,0795 & 0,0098 \\
\hline Fundamental & 0,98 & 1,15 & 1,34 & 0,1373 & 0,0804 & 0,0879 \\
\hline Médio & 0,85 & 0,99 & 1,15 & $-0,0104$ & 0,0776 & 0,8937 \\
\hline Superior (base) & & 1,00 & & & & \\
\hline \multicolumn{7}{|l|}{ Trabalho } \\
\hline Desocupados/inativos (base) & & 1,00 & & & & \\
\hline Ocupados & 1,23 & 1,31 & 1,40 & 0,2713 & 0,0332 & 0,0000 \\
\hline \multicolumn{7}{|l|}{ Renda } \\
\hline $20+$ pobres & 1,88 & 2,12 & 2,39 & 0,7515 & 0,0611 & 0,0000 \\
\hline $20-40$ & 1,53 & 1,71 & 1,92 & 0,5371 & 0,0586 & 0,0000 \\
\hline $40-60$ & 1,43 & 1,59 & 1,77 & 0,4636 & 0,0556 & 0,0000 \\
\hline $60-80$ & 1,20 & 1,33 & 1,48 & 0,2884 & 0,0530 & 0,0000 \\
\hline $20+$ ricos (base) & & 1,00 & & & & \\
\hline \multicolumn{7}{|l|}{ Situação do domicílio } \\
\hline Urbana (base) & & 1,00 & & & & \\
\hline Rural & 1,05 & 1,17 & 1,30 & 0,1558 & 0,0534 & 0,0035 \\
\hline \multicolumn{7}{|l|}{ Região } \\
\hline Norte & 1,64 & 1,89 & 2,19 & 0,6391 & 0,0741 & 0,0000 \\
\hline Nordeste & 1,62 & 1,84 & 2,08 & 0,6073 & 0,0636 & 0,0000 \\
\hline Sudeste & 0,97 & 1,09 & 1,22 & 0,0843 & 0,0599 & 0,1596 \\
\hline Sul (base) & & 1,00 & & & & \\
\hline Centro-Oeste & 1,41 & 1,60 & 1,82 & 0,4726 & 0,0641 & 0,0000 \\
\hline \multicolumn{7}{|l|}{ Estado de saúde } \\
\hline Avaliação negativa & 1,34 & 1,45 & 1,57 & 0,3720 & 0,0408 & 0,0000 \\
\hline Atividades realizadas & 1,22 & 1,32 & 1,44 & 0,2791 & 0,0424 & 0,0000 \\
\hline Não acamado & 1,05 & 1,16 & 1,28 & 0,1476 & 0,0506 & 0,0035 \\
\hline Doença crônica & 0,91 & 0,98 & 1,05 & $-0,0242$ & 0,0351 & 0,4917 \\
\hline Mobilidade limitada & 1,49 & 1,61 & 1,73 & 0,4734 & 0,0373 & 0,0000 \\
\hline \multicolumn{7}{|l|}{ Acesso à saúde } \\
\hline Sem consulta médica & 2,20 & 2,35 & 2,51 & 0,8530 & 0,0336 & 0,0000 \\
\hline Sem cadastro ESF & 1,21 & 1,29 & 1,37 & 0,2513 & 0,0323 & 0,0000 \\
\hline Sem plano de saúde & 2,06 & 2,27 & 2,51 & 0,8206 & 0,0499 & 0,0000 \\
\hline \multicolumn{7}{|l|}{ Outros moradores } \\
\hline Sem necessidade (base) & & 1,00 & & & & \\
\hline Todos c/ necessidade insatisfeita & 5,30 & 5,96 & 6,70 & 1,7855 & 0,0597 & 0,0000 \\
\hline Algum $\mathrm{c} /$ necessidade insatisfeita & 2,68 & 3,09 & 3,57 & 1,1289 & 0,0736 & 0,0000 \\
\hline Todos $\mathrm{c} /$ necessidade satisfeita & 0,63 & 0,67 & 0,72 & $-0,3956$ & 0,0353 & 0,0000 \\
\hline Constante & & & & $-5,2328$ & 0,1132 & 0,0000 \\
\hline
\end{tabular}

Fonte: IBGE, Pesquisa Nacional por Amostra de Domićlios em microdados. (1) Exceto as áreas rurais do Acre, Amapá, Amazonas, Pará, Rondônia e Roraima. (2) Para pessoas de zero a 24 anos que não eram pessoa de referência ou cônjuge no grupo doméstico, considerouse o nível educacional da pessoa de referência. 
mulher de 25 a 44 anos branca e desocupada, sem educação elementar, residindo no Nordeste urbano, entre os $20 \%$ mais pobres da população e sem plano de saúde (com as demais características na base) teria $12,4 \%$ de probabilidade de não buscar atendimento; e um homem com as mesmas características teria $14,5 \%$; e a razão entre as chances correspondentes a essas probabilidades seria 1,2 . Se a mulher fosse preta, parda ou indígena, sua probabilidade subiria para $14,1 \%$.

Estar ocupado faz a chance de não procurar atendimento aumentar 1,3 vezes. A residência em zona rural resulta em chance apenas 1,2 vezes maior. Não ter suspenso atividades nem ter estado acamado (que indicariam uma necessidade "leve", com menor premência pela satisfação) nas duas semanas anteriores implica pequeno aumento da probabilidade de não satisfazer a necessidade de saúde. Pessoas em grupos domésticos não cadastrados pela ESF têm chance pouco maior de ficarem com a necessidade insatisfeita.

Obviamente, muitas dessas características podem ocorrer conjuntamente, elevando bastante o risco dos grupos populacionais que definem. Por exemplo, homens adultos na faixa dos 25 aos 44 anos muito provavelmente estarão ocupados, e se forem pouco educados, também estarão entre os mais pobres e provavelmente não terão plano de saúde - todas essas características somadas levam a um considerável aumento do risco desse grupo.

Outras características têm efeitos maiores. A chance de não buscar atendimento para uma necessidade, de todos os grupos etários, é maior do que a das crianças de zero a quatro anos. Porém, a variação não é linear em respeito à idade. Pessoas de 5 a 24 anos apresentam chances apenas um pouco maiores do que a da categoria de base, mas a chance de não satisfazer a necessidade daquelas de 25 a 44 é o dobro. Nas faixas etárias subseqüentes, a chance é menor do que a da faixa dos 25 aos 44 anos, exceto para as pessoas de 75 ou mais anos.

Residir no Norte, no Nordeste, ou no Centro-Oeste do Brasil aumenta bastante a chance de não buscar atendimento em relação à chance verificada entre os moradores do Sul (ou do Sudeste, cuja chance não é significativamente distinta daqueles dos Sul). E quanto mais pobre é o quinto da distribuição da renda domiciliar per capita em que se está, maior é a chance de não procurar atendimento em relação a uma pessoa que faça parte dos $20 \%$ mais ricos da população.

Também se mostraram relevantes a autoavaliação do estado de saúde e a mobilidade física limitada. Pessoas que avaliam mal a própria saúde têm chance 1,5 vezes maior de não procurar atendimento em caso de necessidade. Mais intuitivo é o fato de que pessoas com limitações de movimentos ou atividades têm chance 1,6 vezes maior de não satisfazerem a necessidade de saúde. O modelo também confirmou a importância dos planos de saúde. Não tê-los, e não ter realizado consultas médicas, são, dentre as características individuais, os dois maiores fatores isolados de risco, fazendo a chance de não procurar atendimento ser 2,3 vezes maior.

Porém, o conjunto de características mais relevantes é o que diz respeito à presença de outros moradores com necessidade de saúde e sua satisfação. Compor um grupo doméstico com outras pessoas que também tiveram necessidade, mas não procuraram atendimento aumenta bastante, 6,0 vezes, a chance de que o próprio sujeito não procure atendimento para a sua necessidade (em relação a uma pessoa idêntica, mas em grupo doméstico no qual, além dela, ninguém teve necessidade). Simplesmente ter mais uma pessoa que não procurou, mesmo na presença de outra que procurou, também aumenta, em 3,1 vezes, a chance de o sujeito não procurar. Por outro lado, conviver somente com pessoas que satisfizeram a própria necessidade reduz a chance de insatisfação.

\section{Discussão}

A análise da necessidade de saúde insatisfeita por causa da não procura de atendimento a partir dos dados dos suplementos de saúde da Pnad revelou que, de 1998 a 2008, a parcela da população total que teve uma necessidade de saúde se manteve em torno de $17 \%$, enquanto a parcela com necessidade de saúde insatisfeita caiu de 3,5\% para 2,9\% (21\% para $17 \%$ da população com necessidade). Esses números não são estritamente comparáveis com os de outros países, por que o período de captação da Pnad é bem mais curto duas semanas - e por causa das diferenças nos questionários e nos níveis de satisfação considerados. Registre-se que a baixa porcentagem não reflete a magnitude do problema: no curto período de captação da pesquisa, são mais de cinco milhões de pessoas com necessidade insatisfeita.

Quanto aos motivos que as pessoas apontam para não satisfazer a necessidade de saúde procurando atendimento, em todos os anos o mais escolhido foi falta de dinheiro. A forte associação entre a renda e a necessidade insatisfeita também é registrada em vários países da Euro$\mathrm{pa}^{13}$ e nos Estados Unidos ${ }^{14}$. 
A proporção dos que escolhiam a falta de dinheiro como justificativa passou de um terço a um quinto de 1998 a 2008, enquanto aumentou a porcentagem dos que alegam problemas do sistema - principal causa de insatisfação do Canadá. Uma possível interpretação dessa mudança seria a de que à medida que o SUS se consolida, expande seus serviços e aumenta seu uso pela população, a insatisfação da necessidade de saúde passa a ser justificada por alegações de falhas do sistema, marcando a passagem de um momento em que as pessoas tinham que confiar mais nos seus próprios recursos para arcar com despesas de saúde para outro em que o Estado adquire maior relevância na provisão de serviços, mas tem dificuldades para fazer frente às demandas com a qualidade requerida pela população. Contudo, não se pode desprezar a possibilidade de as pessoas estarem apontando menos as restrições orçamentárias como motivo de não procura simplesmente por ter crescido substancialmente a renda média das famílias.

Como em outros países, a necessidade insatisfeita não se distribui uniformemente pela população, afetando mais certos grupos. Entre as características que aumentam a propensão a se ter a necessidade de saúde insatisfeita estão algumas das citadas em estudos europeus e norteamericanos: o sexo, a baixa renda, não ter um seguro saúde, ser negro, avaliar mal o próprio estado de saúde e ser adulto. Nessa caracterização dos grupos, os estudos de necessidade estabelecem um diálogo com os estudos de utilização de serviços. Ainda que existam diferenças nos objetivos e objetos dos estudos, as evidências de utilização de serviços de saúde no Brasil (cf. Travassos et al. ${ }^{17}$ ), em geral também apontam desigualdades de acesso e utilização de serviços entre os grupos considerados.

$\mathrm{O}$ fato de os homens terem maior propensão à necessidade insatisfeita é uma idiossincrasia brasileira, pois nos Estados Unidos ${ }^{14}$ e no Canadá ${ }^{15}$ são as mulheres que apresentam maior porcentagem de insatisfação. Essa diferença pode, entretanto, se dever aos conceitos mais abrangentes de necessidade de saúde insatisfeita usados nos estudos desses países. A maior propensão à insatisfação dos negros também foi registrada nos Estados Unidos ${ }^{14}$.

Este resultado se coaduna com as observações de menor tendência à busca de serviços de saúde por parte dos homens. A resistência masculina a procurar médico ou profissional de saúde ao sentir necessidade pode ter fundo cultural e estar assentada nas relações de gênero, incluindo a participação mais elevada no mercado de trabalho. $\mathrm{O}$ fato de homens adultos com idade entre 25 e 44 anos terem maior probabilidade de não satisfazer suas necessidades pode indicar dificuldade de acesso relacionada ao período de funcionamento das unidades de saúde. A combinação dessas características com baixo nível de escolaridade, local de residência (Norte ou Nordeste) e não ter plano de saúde pode ampliar ainda mais o risco desse grupo. Isso também vale para as mulheres ocupadas, mas há proporcionalmente mais homens reunindo simultaneamente várias dessas características que aumentam a chance de insatisfação.

Por fim, o fato de o conjunto de características mais relevantes para a satisfação ou não da necessidade de saúde estar relacionado à presença de outros moradores com necessidade de saúde levanta uma série de hipóteses interessantes. Uma interpretação plausível é a de que devido à homogeneidade cultural e social do grupo doméstico seus membros tomam decisões semelhantes ao confrontarem a necessidade de saúde. Uma pessoa com necessidade pode procurar ou não atendimento a depender do que foi decidido por outras pessoas do mesmo grupo doméstico.

Não obstante, a motivação desse comportamento coletivo do grupo doméstico, em particular o de não procurar atendimento para satisfazer a necessidade de saúde, dificilmente será plenamente compreendida a partir dos dados da Pnad. Não se pode estimar, por exemplo, o peso das barreiras de acesso de ordem individual e o daquelas devidas às insuficiências do sistema de saúde. A elevada importância de se ter plano de saúde para a satisfação da necessidade sugere que as principais barreiras são as insuficiências do sistema. Nesta linha, não ter feito consulta médica no ano anterior indicaria a falta de contato com o sistema de saúde. Mas não se pode excluir a possibilidade de que a razão esteja em circunstâncias individuais, por exemplo, em uma percepção negativa que o grupo doméstico compartilha acerca dos serviços de saúde, ou em sua maioria esses grupos podem ser compostos por casais com ambos os cônjuges ocupados e sem tempo para buscar atendimento no horário regular. São muitas as possibilidades.

Resumindo os resultados à luz do modelo comportamental de Andersen ${ }^{20-21}$, poder-se-ia concluir que fatores de predisposição e fatores capacitantes ainda têm grande peso na determinação da necessidade de saúde insatisfeita. Entre os fatores capacitantes, houve redução relativa da importância da falta de dinheiro e aumento 
da de fatores relacionados ao sistema de saúde. Entre os fatores de predisposição, o sexo, a idade e a condição de ocupação definem grupos com maior propensão a não satisfazer a necessidade de saúde pela procura de atendimento, como demonstram os resultados para homens, ocupados, com idade entre 25 a 44 anos. Ainda que o comportamento do grupo doméstico pareça indicar uma maior importância relativa dos fatores de predisposição, pode de fato estar revelando a percepção ou experiência desse grupo em relação ao acesso e utilização dos serviços de saúde, conforme discutido.

Por ora, as evidências sobre a necessidade de saúde insatisfeita sugerem que se acenda uma luz amarela sobre o grupo dos trabalhadores adultos de baixa renda, em particular os do sexo masculino. Destaque-se que a preocupação, em parte, já encontrou expressão na política pública de saúde, sob a forma do recente programa de atenção à saúde do homem. Mas serão precisos mais estudos sobre a necessidade de saúde insatisfeita pela não procura de atendimento para que se possa obter uma melhor compreensão de suas causas. Seus resultados serão úteis para que, mediante o melhor conhecimento da diversidade de situações, se estabeleçam estratégias específicas de inclusão, com o objetivo de que, conforme previsto na Constituição, o acesso aos serviços de saúde seja efetivamente universalizado.

\section{Colaboradores}

RG Osorio, LMS Servo e SF Piola participaram igualmente de todas as etapas de elaboração do artigo. 


\section{Referências}

1. Campos CMS. Necessidades de saúde pela voz da sociedade civil (os moradores) e do Estado (os trabalhadores de saúde) [tese]. São Paulo (SP): Escola de Enfermagem, Universidade de São Paulo; 2004.

2. Campos CMS, Mishima SM. Necessidades de saúde pela voz da sociedade civil e do Estado. Cad Saude Publica 2005; 21(4):1260-1268.

3. Schraiber LB, Mendes-Gonçalves RB. Necessidades de saúde e atenção primária. In: Schraiber LB, Nemes MIB, Mendes Gonçalves RB, organizadores. Saúde do adulto: programas e ações na unidade básica. São Paulo: Hucitec; 1996. p.29-47.

4. Stotz EN. Necessidades de saúde: mediações de um conceito (contribuição das Ciências Sociais para a fundamentação teórico-metodológica de conceitos operacionais da área de Planejamento em Saúde [tese]. Rio de Janeiro (RJ): Escola Nacional de Saúde Pública, Fundação Oswaldo Cruz; 1991.

5. Asadi-Lari M, Packman C, Gray D. Need for redefining needs. Health and Quality of Life Outcomes 2003; 1(34):1-5.

6. Cavalieri M. Geographical patterns of unmet health care needs in Italy. MPRA, 2009. [acessado 2011 fev]. Disponível em: http:॥mpra.ub.uni-muenchen. de/16097/1/MPRA_paper_16097.pdf

7. Junfang W, Biao Z, Weijun Z, Zhang S, Yinyin W, Chen K. Perceived unmet need for hospitalization service among elderly Chinese people in Zhejiang province J Public Health 2009; 3(4):530-540.

8. Wu Z, Penning MJ, Schimmele CM. Immigrant Status and Unmet Health Needs, Can J Public Health 2005; 96(5):369-373.

9. Alonso J, Orfila F, Ruigòmez A, Ferrer M, Antò J. Unmet health care needs and mortality among Spanish elderly. Am J Public Health 1997; 87(3):365-370.

10. Baggett TP, O'Connell JJ, Singer DE, Rigotti NA. The Unmet Health Care Needs of Homeless Adults: A National Study. Am J Public Health 2010; 100(7):1326-1333;

11. Bitsko RH, Visser SN, Schieve LA, Ross DS, Thurman DJ, Perou R. Unmet health care needs among CSHCN with neurologic conditions. Pediatrics 2009; 124(Supl. 4):S343-S351.

12. Ayanian JZ, Weissman JS, Schneider EC, Ginsburg JA, Zaslavsky AM. Unmet health needs of uninsured adults in the United States. JAMA 2000; 284 (16):2061-2069.
13. Allin S, Masseria C. Unmet need as an indicator of health care access. Eurohealth 2009; 15(3):7-9.

14. Berk ML, Schur CL, Cantor, JC. Ability to obtain health care: recent estimates from the Robert Wood Johnson Foundation National Access to Care Survey. Data Watch. Health Affairs 1995; 14(3):139-146.

15. Sanmartin C, Houle C, Tremblay S, Berthelot, JM. Changes in unmet health care needs. Health Reports 2002; 13(3):15-21.

16. Castro MM, Travassos C, Carvalho MS Fatores associados às internações hospitalares no Brasil. Cien Saude Colet 2002; 7(4):795-811.

17. Travassos C, Viacava F, Pinheiro R, Brito A. Utilização dos serviços de saúde no Brasil: gênero, características familiares e condição social. Rev $\mathrm{Pa}$ nam Salud Publica 2002; 11(5-6):365-373.

18. Aday LA. Andersen R. A framework for the study of access to medical care. Health Services Research 1974; 9:208-220.

19. Andersen R. Revisiting the behavioral model and access to medical care: does it matter? J Health and Social Behavior 1995; 36(1):1-10.

20. Wu Z, Penning, MJ, Schimmele CM. Immigrant Status and Unmet Health Care Needs. Can J of Public Health 2005; 96(5):369-373.

21. Shi L, Stevens GD. Vulnerability and Unmet Health Care Needs: The Influence of Multiple Risk Factors. J Gen Intern Med 2005; 20(2):148-154.

22. Instituto Brasileiro de Geografia e Estatística (IBGE), 2009. Pesquisa nacional por amostra de domicílios 2008, notas metodológicas, pesquisa básica, pesquisa especial de tabagismo e pesquisas suplementares de saúde e acesso à internet e posse de telefone móvel celular para uso pessoal (arquivo que acompanha os microdados).

Artigo apresentado em 06/10/2010

Aprovado em 10/05/2011

Versão final apresentada em 06/07/2011 